\title{
High Esteem and Hurting Others Online: Trait Sadism Moderates the Relationship Between Self-Esteem and Internet Trolling
}

\author{
Evita March, $\mathrm{PhD}^{1}$ and Genevieve Steele, MProfPsych ${ }^{2}$
}

\begin{abstract}
Internet trolling is commonly defined as disruptive online behavior, intended to provoke and distress others for amusement. Previous research has shown that gender (specifically, male), trait psychopathy, and trait sadism significantly predict engaging in trolling. In this study, we sought to replicate and extend previous research by exploring the role of self-esteem in predicting trolling, and possible interactions between self-esteem and personality. Participants $(n=400,67.5$ percent women, average age $=24.97$ years $[S D=8.84])$ completed an online questionnaire, including measures of psychopathy, sadism, self-esteem, and trolling behaviors. Results corroborated previous research showing gender (male) to be a significant predictor of trolling, and trait psychopathy and sadism to be significant positive predictors. Although self-esteem had no additional value on top of trait psychopathy and sadism in explaining trolling, there was a significant interaction between self-esteem and trait sadism. A moderation analysis indicated a positive relationship between self-esteem and trolling, but only when trait sadism was high. These results portray the troll as a callous individual may enjoy causing psychological harm, particularly if their self-esteem is high. These results contribute to building the psychological profile of trolls and provide future directions for research exploring trolling behaviors.
\end{abstract}

Keywords: trolling, gender, self-esteem, psychopathy, sadism

\section{Introduction}

$\mathbf{H}$ IGH ESTEEM AND hurting others online: Trait sadism moderates the relationship between high self-esteem and Internet trolling.

Internet trolling ("trolling") is broadly defined as anonymously causing disruption online by posting provocative content with the intent to trigger conflict and cause victims distress for the "troll's" own amusement ${ }^{1,2}$ Recent research has shown that the general population considers trolling an intentional, provocative, and damaging behavior. ${ }^{3}$ Experiencing trolling has been associated with distress and significant negative psychological outcomes. ${ }^{4,5}$

To explain why people troll, the Social Identity Model of Deindividuation (SIDE model ${ }^{6}$ ) has been applied. ${ }^{7}$ According to the SIDE model, environments that promote deindividuation increase acceptance of group norms while decreasing individual norms. In these deindividuated environments, an individual is more likely to adopt the group norms; for example, an individual who is in a trolling environment is more likely to adopt the group behavior.
Importantly, the SIDE model emphasizes individual differences, and that these individual characteristics could explain why some people, but not others, engage in trolling. In line with the SIDE model emphasizing individual characteristics, the aim of this study is to continue exploring individual differences in perpetrating trolling behaviors.

\section{Predictors of Internet Trolling: Gender and Personality}

Research has shown that men, compared with women, are more likely to perpetrate trolling behaviors. ${ }^{1,8,9}$ In addition to gender, "dark" personality traits, specifically trait psychopathy and trait sadism, show significant utility in predicting trolling behaviors. ${ }^{10}$ Trait psychopathy is characterized by deficits in empathy and self-control, and a deceitful, callous, and unemotional interpersonal style and impulsive behavior. ${ }^{11,12}$ Trait psychopathy has been shown to be a significant positive predictor of trolling, ${ }^{1,7,8}$ with researchers theorizing that the empathic deficits and thrill-seeking characteristics associated with trait psychopathy may lead these individuals to engage in trolling behaviors. ${ }^{9}$

\footnotetext{
${ }^{1}$ School of Health Science and Psychology, Federation University Australia, Berwick, Australia

${ }^{2}$ School of Behavioural and Health Sciences, Australian Catholic University, Melbourne, Australia.
} 
Trait sadism has also been established as a significant positive predictor of trolling. ${ }^{1,7,8,13}$ An individual high in trait sadism derives enjoyment and pleasure from inflicting physical or emotional pain and humiliation onto others. ${ }^{14}$ In a recent experimental study, researchers found a parallel pattern for trolls and individuals with high trait sadism to underestimate others' pain intensity and minimize the suffering of others. ${ }^{13}$ Researchers have even suggested trolling manifests itself in the form of online sadism, and trolls use the Internet as their virtual "playground" to derive enjoyment at the expense of other users'. In sum, this combination of psychopathy and sadism paints the Internet troll as an individual who is callous, lacks empathy, and enjoys causing harm to others. ${ }^{7}$

\section{Self-Esteem and Internet Trolling}

Compared with other online antisocial behavior, such as cyberbullying, trolling remains largely underexplored. Trolling is often confused with cyberbullying ${ }^{9}$ due to shifting definitions of trolling ${ }^{13}$ and sharing similar characteristics, such as aggression. ${ }^{15,16}$ However, although related, researchers posit there are fundamental differences between cyberbullying and trolling ${ }^{17}$ in form, content, intent, and consequence. ${ }^{13}$ For example, deception and meaningless disruption are considered more characteristic of trolling than cyberbullying. ${ }^{15}$ As such, researchers posit that trolling and cyberbullying are distinct antisocial online behaviors. ${ }^{3}$

One trait that has been found to predict other online antisocial behaviors (e.g., cyberbullying), but has not yet been explored as a predictor of trolling, is self-esteem. ${ }^{18}$ Selfesteem is defined as a favorable or unfavorable attitude toward the self, ${ }^{19}$ guided by personal opinions and beliefs. ${ }^{20}$ Researchers suggest that people with low self-esteem shield themselves from feelings of inferiority by projecting blame onto others through hostility and aggression, thus demonstrating antisocial behaviors. ${ }^{21}$ Self-esteem has good predictive utility for engaging (or not engaging) in antisocial behaviors such as aggression, ${ }^{22}$ bullying, ${ }^{23}$ and cyberbullying. ${ }^{24}$ Furthermore, low self-esteem has been associated with deceitful online behaviors, such as creating false identities and lying. ${ }^{25}$

The negative relationship that exists between self-esteem and cyberbullying ${ }^{24}$ and other deceitful behavior online ${ }^{25}$ indicates that someone engaging in this behavior may have low self-worth, and may even experience depression and anxiety. ${ }^{26}$ Indeed, the media has tended to portray the Internet troll as someone with low self-esteem ${ }^{27}$; however, it is unclear what empirical research these claims are based on.

As aggression and deceit are characteristic of trolling, ${ }^{15}$ there is rationale to expect self-esteem to predict perpetration of trolling. Furthermore, self-esteem correlates with the established trolling predictors of gender ${ }^{28}$ and dark personality traits, ${ }^{29}$ thus strengthening the rationale for self-esteem to predict trolling. Finally, as previous research has shown self-esteem significantly interactions with dark personality traits, $^{22,29}$ there is also rationale to expect self-esteem to interact with trait psychopathy and trait sadism to predict perpetration of trolling.

To date, one study ${ }^{17}$ has explored self-esteem and trolling behavior; however, there are some significant concerns regarding this study that limit interpretation and validation of results. First, the authors did not provide a sufficient rationale for creating a new measure of cyberbullying and trolling behaviors (the Cyberbully/Troll Deviancy Scale) when validated measures of the two behaviors already exist. Second, it is unclear why the authors created categories for participants (e.g., cyberbullies only, trolls only, both cyberbullies and trolls, or neither), instead of maintaining the continuous nature of the measure. Third, there were 10 participants in the troll-only category - a sample size unlikely to be a meaningful representation. Finally, and importantly, the authors reported that individuals with low self-esteem were more likely to engage in the troll-only behaviors compared with neither; however, this conclusion is based on results of a chisquare, and thus the utility of self-esteem in predicting trolling is unknown.

Based on the limitations apparent in this study, combined with the rationale to expect utility to expect self-esteem to predict trolling, the aim of this study was to explore the utility of gender, trait psychopathy, trait sadism, and selfesteem in predicting perpetration of trolling. It was predicted that (male) gender, (high) trait psychopathy, and (high) trait sadism would predict higher perpetration of trolling behaviors, and self-esteem would explain additional variance. Finally, as previous research has shown self-esteem significantly interacts with dark personality traits, it was predicted that self-esteem would significantly interact with trait psychopathy and trait sadism to predict trolling.

\section{Method}

\section{Participants and procedure}

Participant recruitment commenced upon receipt of Human Research Ethics Committee from the relevant institution. Potential participants were recruited through Facebook and Reddit advertisements that directed them to an online anonymous confidential questionnaire. The only inclusion criterion was aged $\geq 18$ years. The final ${ }^{\mathrm{a}}$ convenience sample consisted of 400 participants (67.5 percent women, 32.5 percent men). Participants were aged between 18 and 75 years, with a mean age of 24.97 years $(S D=8.84)$. The majority of participants reported their nationality as Australian (43.3 percent), spent 1-2 hours online per day (29.6 percent), were currently employed (73.7 percent), and were currently completing an undergraduate degree (59.5 percent).

\section{Measures}

The Global Assessment of Internet Trolling-Revised (GAIT-R ${ }^{9}$ ) was used to assess trolling behaviors. Participants indicated their responses to 8-items (e.g., "I enjoy upsetting people I do not personally know on the Internet"; current Cronbach's alpha $=0.81$ ) measured on a 5-point Likert scale ( $1=$ strongly disagree, $5=$ strongly agree). Responses to items are summed for a total score, with higher scores indicating more perpetration of trolling behaviors.

Trait psychopathy was measured with the psychopathy subscale of the 27-item Short Dark Triad scale (SD3 $\left.{ }^{30}\right)$. The full SD3 was administered to participants; however, only trait psychopathy was calculated for study aims and hypotheses. The psychopathy subscale includes nine items ("I like to get revenge on authorities"; current Cronbach's alpha= 0.72) and participants indicated agreement to statements on a 5 -point Likert scale ( $1=$ strongly disagree, $5=$ strongly agree). 
Table 1. Descriptives ( $M$ and $S D$ ) and Bivariate Pearson Correlations for Variables of Gender, Psychopathy, Sadism, Self-Esteem, and Trolling Behaviors

\begin{tabular}{|c|c|c|c|c|c|}
\hline Variable & $\mathrm{M}(\mathrm{SD})$ & 1 & 2 & 3 & 4 \\
\hline 1. Gender & - & - & & & \\
\hline 2. Psychopathy & $18.90(5.01)$ & $-0.24 * *$ & - & & \\
\hline 3. Sadism & $15.62(5.57)$ & $-0.18 * *$ & $0.53 * *$ & - & \\
\hline 4. Self-esteem & $28.77(5.75)$ & -0.03 & $-0.22 * *$ & $-0.28 * *$ & - \\
\hline 5. Trolling & $13.18(4.92)$ & $-0.34 * *$ & $0.56 * *$ & $0.59 * *$ & $-0.11 *$ \\
\hline
\end{tabular}

Gender is coded as men $=0$, women $=1$; correlations corrected for multiple testing. $* p<0.05$.

$* * p<0.001$.

Trait sadism was measured using the Short Sadistic Impulse Scale. ${ }^{31}$ Participants responded to 10-items (e.g., "I enjoy seeing people hurt'; current Cronbach's alpha $=0.84$ ) on a 5 -point Likert scale $(1=$ strongly disagree, $5=$ strongly agree).

Individual self-esteem was measured with the Rosenberg Self-Esteem Scale. ${ }^{19}$ Participants responded to 10-items (e.g., "I feel that I have a number of good qualities"; current Cronbach's alpha $=0.91$ ) on a 4-point Likert scale ( $1=$ strongly disagree, $4=$ strongly agree $)$.

\section{Design}

The design of the study was correlational, with the predictor variables of gender, trait psychopathy, trait sadism, self-esteem, and interactions between trait psychopathy and self-esteem and trait sadism and self-esteem as predictors. Internet trolling was the outcome variable. Based on the large age range of the sample (18-75 years), age was included in the analyses as a control variable.

\section{Results}

Descriptives and bivariate Pearson correlations between predictor variables of gender, psychopathy, sadism, and self- esteem and the outcome variable of Internet trolling are presented in Table 1.

Significant correlations between all predictors and criterion support their inclusion in a regression model. A two-step hierarchical regression analysis was performed to predict Internet trolling, with gender, psychopathy, and sadism entered at Step 1. Age was also included in Step 1 as a control variable. Self-esteem and interactions between self-esteem, psychopathy, and sadism were entered at Step 2. $F$ tests, significance, adjusted $R^{2}$, and coefficient statistics can be seen in Table 2.

Table 2 shows that at Step 1, age was not a significant predictor of trolling. Gender (specifically, male) was a significant predictor, and trait psychopathy and sadism were significant positive predictors of trolling. At Step 2, gender (male) was a significant predictor of trolling, and psychopathy and sadism were significant positive predictors of trolling. There was also a significant interaction between selfesteem and sadism. This interaction was explored using a PROCESS moderation analysis with sadism moderating the relationship between self-esteem and trolling (Table 3 ).

Results indicated a significant positive relationship between self-esteem and trolling, but only when sadism was high. Figure 1 displays the graphed moderation analysis.

Table 2. Hierarchical Multiple Regression Analysis with Gender, Psychopathy, Sadism, Self-Esteem, and Interactions as Predictors and Internet Trolling as the Criterion

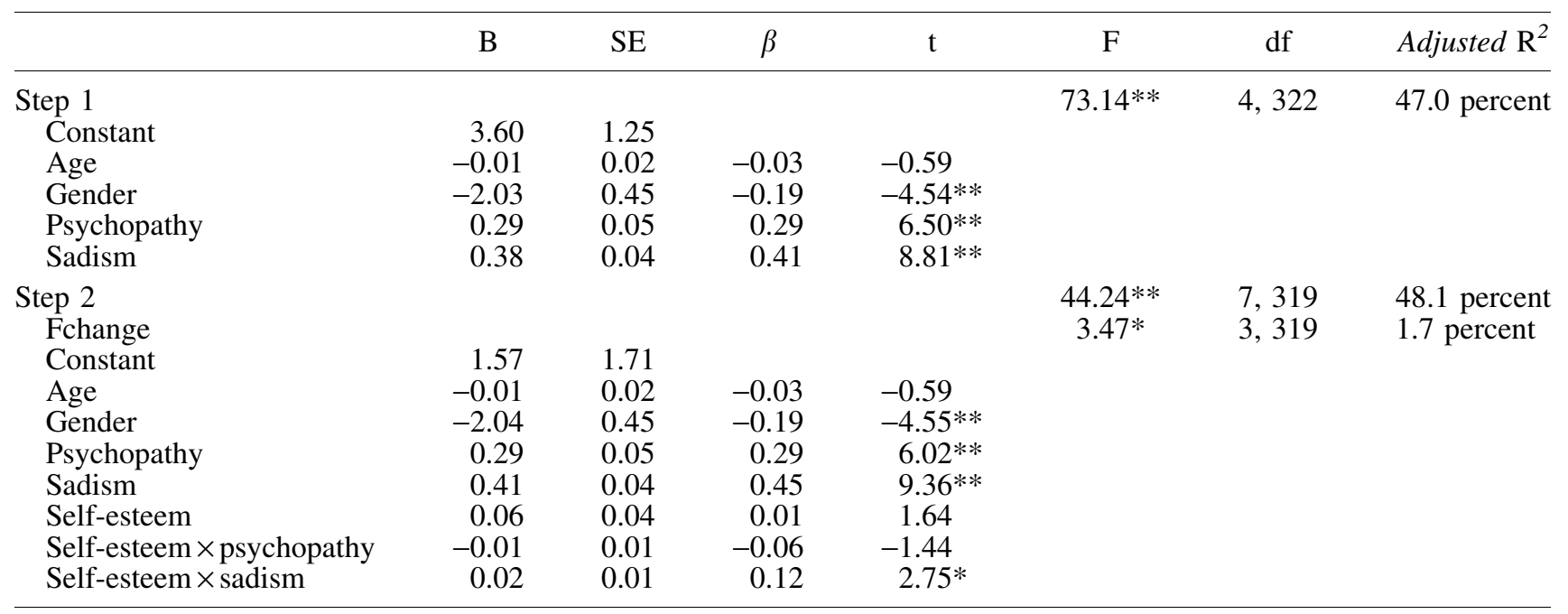

Gender is coded as men $=0$, women $=1$.

$* p<0.01$.

$* * p<0.001$. 
Table 3. Conditional Effects of Self-Esteem on Trolling at low, Medium, and High Levels OF SELF-EsteEM

\begin{tabular}{lrcc}
\hline & Effect & SE & $\mathrm{t}$ \\
\hline Trolling & & & \\
Low sadism & -0.05 & 0.05 & -1.01 \\
Medium sadism & 0.03 & 0.04 & 0.65 \\
High sadism & 0.18 & 0.06 & $2.92^{*}$ \\
\hline
\end{tabular}

Effect refers to effects of $\mathrm{X}$ on $\mathrm{Y}$ at $-1 S D, 0 S D$, and $+1 S D$ of the moderator.

$* p<0.05$.

SE, standard error.

\section{Discussion}

The aim of this study was to explore the utility of gender, trait psychopathy, trait sadism, and self-esteem in predicting perpetration of trolling. Results partially supported the hypotheses, indicating gender (male), (high) trait psychopathy, and (high) trait sadism were significant predictors of trolling; however, self-esteem has no additional value on top of trait psychopathy and sadism in explaining trolling. Furthermore, there was a significant interaction between trait sadism and self-esteem; at high levels of sadism, there was a significant positive relationship between self-esteem and trolling.

\section{Internet trolling: gender, psychopathy, and sadism}

The current result regarding gender corroborates results of previous research ${ }^{1,9}$ and provides support to the premise that men, compared with women, are more likely to engage in online antisocial behaviors. ${ }^{32-34}$ These gender differences in online antisocial behavior may be a reflection of gender stereotypes, where agentic characteristics such as competitiveness and dominance are encouraged in men. ${ }^{35}$

Trait psychopathy and sadism were both significant positive predictors of trolling, corroborating results of previous research ${ }^{7,13}$ and providing further evidence for these traits to reliably predict trolling behaviors. The thrill-seeking nature of the individual high on trait psychopathy could enjoy the thrill of causing online social mayhem through trolling. 9 Furthermore, the empathy deficits and deceitful interpersonal style characteristic of trait psychopathy ${ }^{11}$ aligns with the deception of trolling and the callous unprovoked attacks on other online users. 18

FIG. 1. PROCESS analysis for sadism moderating relationship between self-esteem trolling. Y axis starts at score of 9.
Regarding trait sadism and trolling, individuals with high trait sadism derive pleasure from causing pain to others, thus reflecting the enjoyment trolls experience inflicting suffering and humiliation on their victims. ${ }^{2,13}$ Not only do individuals with high trait sadism enjoy harming others, but they are willing to endure personal strain and sacrifice just for the opportunity to do so. ${ }^{36}$ This behavior is also characteristic of trolls, who invest a significant amount of time and energy into anonymously disrupting and destroying online spaces. ${ }^{13}$ The cloak of anonymity the online space offers allows trolls to enact on normally inhibited sadistic impulses. Trolls use this online anonymity to inflict pain onto others, deriving pleasure and enjoyment from their suffering.

\section{Internet trolling and self-esteem}

Despite predictions, results of this study indicated selfesteem had no additional value on top of trait psychopathy and sadism in explaining trolling. This result does not support previous chi-square analyses that individuals with low self-esteem were more likely to engage in troll-only behaviors. ${ }^{17}$ Furthermore, this result does not support negative relationships found between self-esteem and other online antisocial behaviors, such as cyberbullying. ${ }^{24}$

The lack of utility for self-esteem to predict trolling is contrary to media conceptualizations of trolls attributing this behavior to low self-esteem. ${ }^{27,37}$ It is unclear why, in the absence of reliable scientific findings, the media has attributed trolling to low self-esteem. One possibility is that trolling is often inaccurately conceptualized as a form of cyberbullying, 9,13 and the media may have generalized the relationship between low self-esteem and cyberbullying to include trolling. Another possibility is that by portraying the troll as someone with low self-esteem, this "humanizes" the troll by attributing their callous unpleasant online behavior to a lack of positive self-regard and esteem. Despite these media claims, results of this study do not contribute to profiling the Internet troll as someone with low self-worth.

\section{Interaction between self-esteem and sadism}

Although self-esteem did not uniquely predict trolling, there was a significant interaction between self-esteem and trait sadism. A moderation analysis indicated higher levels of individual self-esteem predicted trolling, but only when

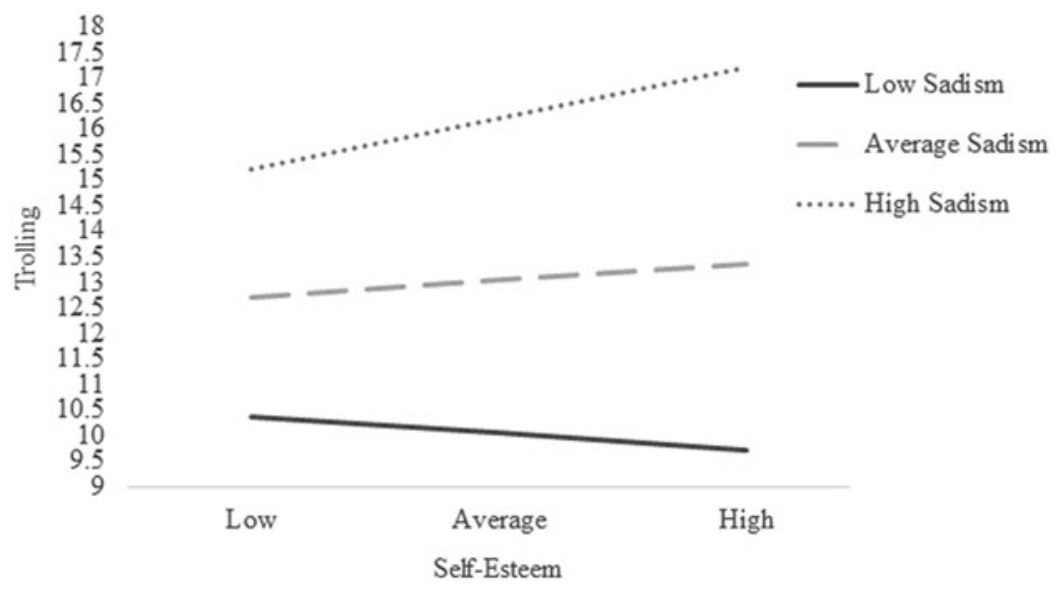


the individual also had high levels of trait sadism. Given the evidence linking low self-esteem to aggressive behaviors, ${ }^{21,22,38}$ for individuals with high levels of trait sadism the positive relationship between self-esteem and trolling was initially surprising. Still, a pattern between higher selfesteem and aggressive behaviors has also been demonstrat$\mathrm{ed}^{39,40}$; however, researchers note that this pattern is more likely attributed to higher levels of narcissism, which is better correlated with high (not low) self-esteem. ${ }^{41}$

As we used the $\mathrm{SD}^{30}$ in this study to measure psychopathy, we subsequently collected data to measure trait narcissism. To test if the positive relationship between selfesteem and trolling (an aggressive behavior) is perhaps better attributed to narcissism masking itself as self-esteem, we conducted an additional moderation analysis ${ }^{\mathrm{b}}$ with trait narcissism predicting trolling and sadism as the moderator and a similar pattern of results was observed. This indicates two important outcomes: (a) the component of self-esteem that predicts engaging in trolling behaviors may bear similarity to narcissism, and (b) this component is only predictive of trolling when an individual enjoys causing psychological and physical harm to others.

The positive relationship between esteem (or egoism) and trolling being dependent on higher levels of trait sadism indicates that an individual is more likely to troll if they have a favorable attitude toward themselves combined with a desire to inflict pain unto and dominate others. If individuals feel good about themselves (i.e., has high esteem), and want to hurt and shame others (i.e., high sadism), they are more inclined to engage in trolling.

\section{Limitations and future research}

Despite the contribution to research on trolling the current project offers, results must be considered in the context of possible limitations. One potential limitation is that the research relied exclusively on self-report data, and possible response bias might exist when assessing antisocial personality traits and online behavior. We recommend future research exploring online antisocial behaviors endeavor to overcome limitations associated with self-report by developing more objective measures of online behavior.

Future research should also seek to continue exploring the interaction between esteem/egoism, sadism, and antisocial online behavior. A possible direction is differentiating between grandiose and vulnerable narcissism, as grandiose (not vulnerable) narcissism is associated with self-esteem, ${ }^{42}$ and might be better associated with aggressive behaviors. ${ }^{43,44}$ To explore the covariance between self-esteem and narcissism in predicting antisocial online behavior, and to determine if this relationship is better attributed to grandiose or vulnerable narcissism, future research could include measures of grandiose narcissism and vulnerable narcissism (such as the Brief-Pathological Narcissism Inventory ${ }^{45}$ ).

Finally, future research should seek to establish the specific characteristics of psychopathy that predict trolling. Exploring characteristics of psychopathy (e.g., empathy, impulsivity, and thrill-seeking behavior) as individual predictors may elucidate exactly which components of trait psychopathy predict this behavior, thus providing further insight into the relationship between antisocial characteristics and Internet trolling ${ }^{c}$.

\section{Conclusion}

The current research adds to the growing body of literature exploring predictors of Internet trolling and has further substantiated explaining trolling through individual characteristics, as emphasized by the SIDE model. These results have corroborated previous findings regarding gender, psychopathy, and sadism, and have extended previous research by providing new evidence regarding the predictive utility of self-esteem. Although self-esteem had no additional value on top of trait psychopathy and sadism in explaining trolling, self-esteem did significantly interact with trait sadism. Contrary to media conceptualizations of the troll as an individual with low self-esteem, results of this study indicate that an individual with higher esteem might be more inclined to troll, particularly if they enjoy causing psychological harm.

\section{Notes}

a. An initial 528 participants accessed the questionnaire link.

b. This moderation analysis is available upon request.

c. Thank you to an anonymous reviewer for this recommendation.

\section{Author Disclosure Statement}

No competing financial interests exist.

\section{Funding Information}

This study received no funding.

\section{References}

1. Buckels EE, Trapnell, PD, Paulhus DL. Trolls just want to have fun. Personality and Individuals Differences 2014; 67 : 97-102.

2. Kopecky K. Misuse of web cameras to manipulate children with the so-called webcam trolling. Telematics and Informatics 2016; 33:1-7.

3. March E, Marrington JZ. Qualitative analysis of Internet Trolling. Cyberpsychology, Behavior, and Social Networking 2019; 22:192-197.

4. Coles B, West M. Trolling the trolls: online forum users constructions of the nature and properties of trolling. Computers in Human Behaviour 2016; 60:233-244.

5. Thacker S, Griffiths M. An exploratory study of trolling in online video gaming. International Journal of Cyber Behavior, Psychology, and Learning 2012; 2:17-33.

6. Reicher S, Spears R, Postmes T. A social identity model of deindividuation phenomena. European Review of Social Psychology 1995; 6:161-198.

7. March E. Psychopathy, sadism, empathy, and the motivation to cause harm: new evidence confirms malevolent nature of the Internet troll. Personality and Individual Differences 2019; 141:133-137.

8. Craker N, March E. The dark side of Facebook ${ }^{\circledR}$ : the Dark Tetrad, negative social potency, and trolling behaviours. Personality and Individual Differences 2016; 102:79-84.

9. Sest N, March E. Constructing the cyber-troll: psychopathy, sadism, and empathy. Personality and Individual Differences 2017; 119:69-72.

10. March E, Grieve R, Marrington JZ, et al. Trolling on Tinder (and other dating apps): examining the role of the Dark 
Tetrad and impulsivity. Personality and Individual Differences 2017; 110:139-143.

11. Pabian S, De Backer C, Vandebosch H. Dark Triad personality traits and adolescent cyber-aggression. Personality and Individuals Differences 2015; 75:41-46.

12. Paulhus DL, Williams KM. The Dark Triad of personality: narcissism, Machiavellianism, and psychopathy. Journal of Research in Personality 2002; 36:556-563.

13. Buckels EE, Trapnell PD, Andjelovic T, et al. Internet trolling and everyday sadism: parallel effects on pain perception and moral judgment. Journal of Personality 2019; 87:328-340.

14. Mededovic J, Petrovic B. The Dark Tetrad: structural properties and location in the personality space. Journal of Individual Differences 2015; 36:228-236.

15. Hardaker C. Trolling in aysnchronous computer-mediated communication: from user discussions to academic definitions. Journal of Politeness Research 2010; 6:215-242.

16. Dooley JJ, Pyżalski J, Cross D. Cyberbullying versus faceto-face bullying: a theoretical and conceptual review. Zeitschrift für Psychologie/Journal of Psychology 2009; 217:182-188.

17. Zezulka LA, Seigfried-Spellar KC. Differentiating cyberbullies and Internet trolls by personality characteristics and self-esteem. Journal of Digital Forensics, Security and Law 2016; 11:7-26.

18. Gibb ZG, Devereux PG. Who does that anyway? Predictors and personality correlates of cyberybullying in college. Computers in Human Behavior 2014; 38:8-16.

19. Rosenberg M. (1965) Society and the adolescent selfimage. New Jersey: Princeton University Press.

20. Baranik LE, Meade AW, Lakey CE, et al. Examining the differential item functioning of the Rosenberg self-esteem scale across eight countries. Journal of Applied Social Psychology 2008; 38:1867-1904.

21. Tracy JL, Robins RW. "Death of a (narcissistic) salesman": an integrative model of fragile self-esteem. Psychological Inquiry 2003; 14:57-62.

22. Donnellan MB, Trzesniewski KH, Robins RW, et al. Low self-esteem is related to aggression, antisocial behavior, and delinquency. Psychological Science 2005; 16:328-335.

23. Fanti KA, Henrich CC. Effects of self-esteem and narcissism on bullying and victimization during early adolescence. The Journal of Early Adolescence 2015; 35:5-29.

24. Patchin JW, Hinduja S. Cyberbullying and self-esteem. Journal of School Health 2010; 80:614-621.

25. Harman JP, Hansen CE, Cochran ME, et al. Liar, liar: Internet faking but not frequency of use affects social skills, self-esteem, social anxiety, and aggression. CyberPsychology \& Behavior 2005; 8:1-6.

26. Mann MM, Hosman CM, Schaalma HP, et al. Self-esteem in a broad-spectrum approach for mental health promotion. Health Education Research 2004; 19:357-372.

27. Taylor E. (2012) Internet trolls 'have low self esteem'. http://www.stuff.co.nz/life-style/7245308/Internet-trolls-havelow-self-esteem (accessed August 1, 2019).

28. Kling KC, Hyde JS, Showers CJ, et al. Gender differences in self-esteem: a meta-analysis. Psychological Bulletin 1999; 125:470-500.

29. Brookes J. The effect of overt and covert narcissism on self-esteem and self-efficacy beyond self-esteem. Personality and Individuals Differences 2015; 85:172-175.

30. Jones DN, Paulhus DL. Introducing the Short Dark Triad (SD3): a brief measure of dark personality traits. Assessment $2014 ; 21: 28-41$.
31. O'Meara A, Davies J, Hammond S. The psychometric properties and utility of the short sadistic impulse scale (SSIS). Psychological Assessment 2011; 23:523-531.

32. Barlett C, Coyne SM. A meta-analysis of sex differences in cyber-bullying behaviour: the moderating role of age. Journal of Aggressive Behaviour 2014; 40:1098-2337.

33. Erdur-Baker O. Cyber-bullying and its correlation to traditional bullying, gender and frequent and risky usage of internet-mediated communication tools. New Media \& Society 2009; 12:109-125.

34. Festl R, Quandt TJ. The role of online communication in long-term cyberbullying involvement among girls and boys. Journal of Youth Adolescence 2016; 45:1931-1945.

35. Feinstein BA, Hershenberg R, Bhatia V, et al. Negative social comparison of Facebook and depressive symptoms: rumination as a mechanism. Psychology of Popular Media Culture 2013; 2:161-170.

36. Buckels EE, Jones DN, Paulhus DL. Behavioural confirmation of everyday sadism. Psychological Science 2013; 24:2201-2209.

37. Exploringyourmind. (2019) Internet trolls and their aggression. Retrieved from https://exploringyourmind.com/ internet-trolls-aggression/ (accessed August 1, 2019).

38. Falkenbach D, Howe J, Falki M. Using self-esteem to disaggregate psychopathy, narcissism, and aggression. Personality and Individual Differences 2013; 54:815-820.

39. Salmivalli C. Feeling good about oneself, being bad to others? Remarks on self-esteem, hostility, and aggressive behavior. Aggression and Violent Behavior 2001; 6:375393.

40. Thomaes S, Bushman BJ, Stegge H, et al. Trumping shame by blasts of noise: narcissism, self-esteem, shame, and aggression in young adolescents. Child Development 2008; 79:1792-1801.

41. Ostrowsky MK. Are violent people more likely to have low self-esteem or high self-esteem? Aggression and Violent Behavior 2010; 15:69-75.

42. Zajenkowski M, Maciantowicz O, Szymaniak K, et al. Vulnerable and grandiose narcissism are differentially associated with ability and trait emotional intelligence. Frontiers in Psychology 2018; 9:1606.

43. Lobbestael J, Baumeister RF, Fiebig T, et al. The role of grandiose and vulnerable narcissism in self-reported and laboratory aggression and testosterone reactivity. Personality and Individual Differences 2014; 69:22-27.

44. Pincus A, Ansell E, Pimentel C, et al. Initial construction and validation of the pathological narcissism inventory. Psychological Assessment 2009; 21:365-379.

45. Schoenleber M, Roche MJ, Wetzel E, et al. Development of a brief version of the Pathological Narcissism Inventory. Psychological Assessment 2015; 27:1520-1526.

Address correspondence to: Dr. Evita March School of Health Science and Psychology Federation University Australia Berwick Campus Berwick 3806 Victoria Australia

E-mail: e.march@federation.edu.au 